\title{
A formulation for optimal design problem of compliant displacement magnification mechanisms based on effective energy concept
}

\author{
Takayuki YAMADA*, Kazuhiro IZUI* and Shinji NISHIWAKI* \\ * Department of Mechanical Engineering and Science, Kyoto University \\ C3, Kyotodaigaku-katsura, Nishikyo-ku, Kyoto, 615-8540, Japan \\ E-mail: takayuki@me.kyoto-u.ac.jp
}

Received: 7 September 2017; Revised: 6 October 2017; Accepted: 27 October 2017

\begin{abstract}
A compliant mechanism is a structure designed to be flexible in order to achieve a specified motion as a mechanism. Such mechanisms can enable a variety of functions. In particular, the operation of highly sensitive sensors and actuators in industrial applications often requires reliable and flexible displacement magnification mechanisms. It is well known that topology optimization offers a useful and powerful method for compliant mechanism design, where displacement in a targeted direction at an output port is to be maximized based on the use of artificial spring components located at the boundaries of the input and output ports. However, such spring components are not always a fundamental requirement. This letter presents a formulation for a compliant displacement magnification mechanism design based on the concept of effective energy. Several numerical examples are provided to confirm the utility of the proposed formulation.
\end{abstract}

Keywords : Topology optimization, Compliant mechanisms, Energy effective concept, Displacement magnification mechanisms, Optimum Design

\section{Introduction}

Compliant mechanisms are specially designed structures whose flexibility enables a targeted deformation. Compliant mechanisms typically have fewer mechanical parts than conventional mechanisms, need no lubrication, are nearly silent in operation, and can often be miniaturized, so they are therefore widely used in a variety of products (Howell, 2001). As a type of compliant mechanism, devices that magnify displacement are particularly important in the design of highly sensitive sensors. Although a kinematic synthesis approach is typically used, in which rigid-body mechanism are synthesized, these methods yield lumped mechanism designs (Her and Midha 1987) that may not be optimal. That is, although the design is a solution that satisfies the design requirements, other design solutions may provide higher performance.

To overcome this issue, a topology optimization method (Bendsøe and Kikuchi 1988) has often been applied in compliant mechanism design problems. The first and most significant work was that of Sigmund 1997, in which the objective function is set as the mechanical advantage (MA) (Salamon and Midha 1998), the ratio of input and output forces. On the other hand, Kota et al. 2001 formulated an optimization problem using the geometrical advantage (GA), the ratio of input and output displacements. In their approach, the objective function is set as the GA divided by the total strain energy of the input force so that the GA and stiffness are maximized for a given input force. Frecker et al. 1997 and Nishiwaki et al. 1998 formulated the flexibility of compliant mechanisms using the concept of mutual energy, with sufficient stiffnesses considered through the use of mean compliances.

Topology optimization methods have been increasingly popular in research on optimal design problems for compliant mechanisms and actuators, with the objective function usually set to maximize output displacement and spring components set on input and output boundaries to provide implicit stiffness. This approach has been extended to many applications, such as multi-physics actuator design problems (Sigmund 2001, Yamada et al. 2011), and the standard formulation and design settings for a compliant force inverter are well known as a benchmark topology optimization model 
(Wang et al. 2011, Sigmund 2007). Although this standard formulation can be widely applied when designing compliant devices, it offers no particular advantage in the design of compliant displacement magnification mechanisms.

The aim of this letter is to formulate an optimal design problem for a compliant mechanism that provides more effective displacement magnification, based on the concept of effective energy. The addition of spring components at the input and output boundaries is not required in our formulation. In the next section, we briefly discus the compliant mechanism design problem, introduce a standard formulation, and formulate the design problem. Numerical examples are then provided, using a benchmark model to confirm the effectiveness of the proposed formulation, followed by a brief conclusion.

\section{Formulation}

We consider a domain $\Omega$ for a compliant mechanism, filled with a homogeneous isotropic linearly elastic solid and fixed on boundary $\Gamma_{u}$, as shown in Fig. 1. We restrict the distribution of the material domain $\Omega$ in the fixed design domain

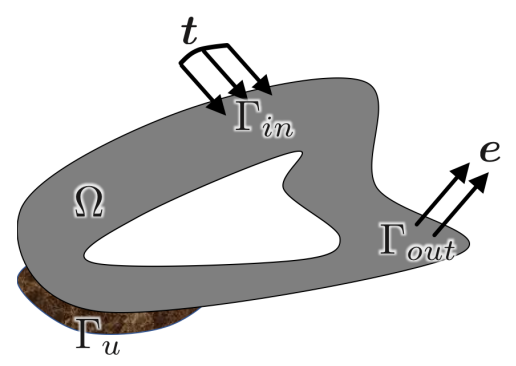

Fig. 1 Concept of compliant mechanisms

$D$. The design variable represents the configuration of the material domain, $\Omega$. The design requirement for this compliant mechanism is to transform traction $t$ on the input boundary $\Gamma_{\text {in }}$ into a deformation at output boundary $\Gamma_{\text {out }}$ in the prescribed direction $\boldsymbol{e}$.

\subsection{Standard formulation}

In the standard formulation (Wang et al. 2011, Sigmund 2007), spring components are set on the input boundary $\Gamma_{i n}$ and output boundary $\Gamma_{\text {out }}$, and the objective function $F_{s}$ is set to maximize output displacement in the prescribed direction $\boldsymbol{e}$, as follows:

$$
\begin{array}{rrr}
\max _{\Omega} & F_{s}:=\int_{\Gamma_{\text {out }}} \boldsymbol{e} \cdot \boldsymbol{u} \mathrm{d} \Gamma & \\
\text { s.t.: } G_{1} & :=-\operatorname{div}\left\{\frac{1}{2} \boldsymbol{C}\left(\nabla \boldsymbol{u}+(\nabla \boldsymbol{u})^{T}\right)\right\}=0 & \text { in } \Omega \\
\boldsymbol{u} & =0 & \text { on } \Gamma_{u} \\
\sigma_{\boldsymbol{n}} & =-k_{\text {out }} \boldsymbol{u} & \text { on } \Gamma_{\text {out }} \\
\sigma_{\boldsymbol{n}} & =\boldsymbol{t}-k_{\text {in }} \boldsymbol{u} & \text { on } \Gamma_{\text {in }} \\
G_{2} & :=\frac{\int_{\Omega} \mathrm{d} \Omega}{\int_{D} \mathrm{~d} \Omega}-V_{\max } \leq 0 &
\end{array}
$$

where $\boldsymbol{u} \in H^{1}(\Omega)$ is the displacement, $\sigma_{n}$ is the traction force, and $\boldsymbol{C}$ is the elastic tensor. $k_{\text {out }}$ and $k_{\text {in }}$ are spring constants on boundaries $\Gamma_{\text {out }}$ and $\Gamma_{\text {in }}$, respectively, and $V_{\max }$ is the upper limit of the volume constraint, $G_{2}$. In the standard formulation, the spring component at the output boundary provides implicit stiffness against reaction forces from the workpiece, which makes the value of spring constant $k_{\text {out }}$ especially significant from an engineering standpoint.

\subsection{Formulation of effective energy concept}

Using a different approach, we propose a different formulation based on the concept of effective energy, in which the compliant mechanism is considered as a device for energy transmission from input force to the target deformation. The objective function, $F_{p}$, is formulated to maximize the energy effectiveness with respect to the target deformation as 
follows:

$$
\begin{array}{lll}
\max _{\Omega} & F_{p}=\frac{U_{o}}{E} & \\
\text { s.t.: } & G_{1}=0 & \text { in } \Omega \\
& \boldsymbol{u}=0 & \text { on } \Gamma_{u} \\
& \sigma_{\boldsymbol{n}}=\boldsymbol{t} & \text { on } \Gamma_{i n} \\
& G_{2} \leq 0, &
\end{array}
$$

where $E$ and $U_{o}$ are the input energy and averaged displacement at the output boundaries, respectively, defined as

$$
\begin{aligned}
E & :=\frac{1}{2} \int_{\Gamma_{\text {in }}} \boldsymbol{t} \cdot \boldsymbol{u} \mathrm{d} \Gamma \\
U_{o} & :=\frac{\int_{\Gamma_{\text {out }}} \boldsymbol{e} \cdot \boldsymbol{u} \mathrm{d} \Gamma}{\int_{\Gamma_{\text {out }}} \mathrm{d} \Gamma} .
\end{aligned}
$$

The objective function here maximizes the target deformation with an effectiveness that depends on the input energy. The resulting values of input energy $E$ are different for different shapes because traction $t$ is constant. The objective function in the standard formulation does not consider changes in input energy corresponding to changes in the shape of $\Omega$, so the effectiveness with respect to input energy is ignored.

In the proposed formulation, the required stiffness as a device is implicitly provided by minimizing the input energy, because such minimization is equivalent to maximization of the stiffness with respect to an input force. Although the characteristics of the objective function here are essentially equivalent to the GA in a linear system, the meaning and concept of settings are different than when the GA is to be maximized. Furthermore, stiffness is not directly considered in our formulation. Naturally, spring components should be set at the output port if there is any reaction force from the workpiece. Kota et al. 2001 considered the GA while maximizing the strain energy for an input force, to directly preserve sufficient stiffness as a structure.

\section{Numerical Examples}

Numerical examples are now provided to confirm the utility of the proposed formulation. Figure 2 shows the fixed design domain and boundary conditions for the standard benchmark model of a compliant mechanism (Sigmund 2007). The fixed design domain is discretized using a structural mesh and four-node quadrilateral plane stress elements with

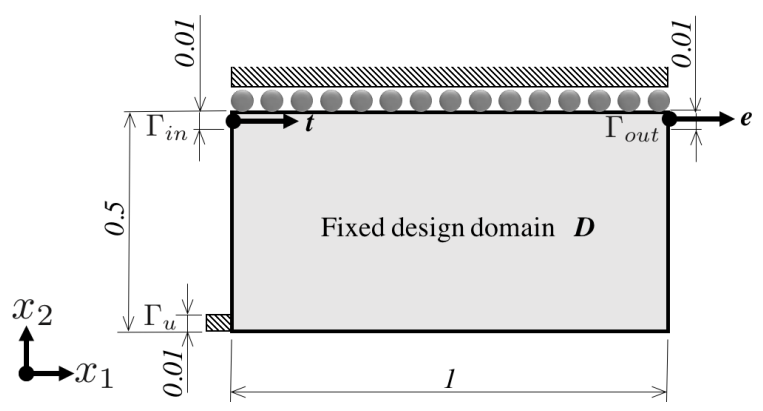

Fig. 2 Fixed design domain and boundary conditions

length $1 \times 10^{-2}$. Young's modulus for the material domain and the fictitious weak material domain are set to 1 and $1 \times 10^{-3}$, respectively. Poisson's ratio, the thickness of the fixed design domain, and the upper limit of the volume constraint are set to $0.31,0.01$, and 0.3 , respectively. The traction force is set so that $t=\left(\begin{array}{ll}1 & 0\end{array}\right)^{T}$, and six boundary condition cases are considered, as indicated in Tab. 3.

We note that case (a) is the compliant inverter with spring components (Wang et al. 2001, Sigmund 2007). Here, we use the Solid Isotropic Material with Penalization (SIMP) method with the Method of Moving Asymptotes (MMA) (Svanberg 1987) and a level set-based topology optimization method (Yamada et al. 2010, Otomori et al. 2015). Figures 3 , 4, 5 and 6 show the obtained material distributions and resulting output displacement $U_{o}$ values, as well as the GA $U_{o} / U_{i}$ when using the standard formulation and the proposed formulation, respectively. That is, the objective functions are set to $F_{s}$ and $F_{p}$, respectively. 
Table 1 Design problem parameters

\begin{tabular}{cccll}
\hline case & \multicolumn{2}{c}{$\boldsymbol{e}$} & $k_{\text {in }}$ & $k_{\text {out }}$ \\
\hline (a) & $(-1$ & $0)^{T}$ & 1 & 0.001 \\
\hline (b) & $(-1$ & $0)^{T}$ & 0 & 0.001 \\
\hline (c) & $(-1$ & $0)^{T}$ & 0 & 0 \\
\hline (d) & $(1$ & $0)^{T}$ & 1 & 0.001 \\
\hline (e) & $(1$ & $0)^{T}$ & 0 & 0.001 \\
\hline (f) & $(1$ & $0)^{T}$ & 0 & 0 \\
\hline
\end{tabular}
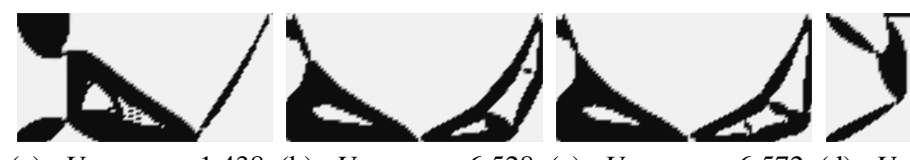

(a) $U_{o}=1.43$
$U_{o} / U_{i}=2.463$

$1.438,(\mathrm{~b}) \quad U_{o}=$
$U_{o} / U_{i}=0.465$

$6.528,(\mathrm{c}) U_{o}=$
$U_{o} / U_{i}=0.367$

$6.572,(\mathrm{~d}) \quad U_{o}=$

$U_{o} / U_{i}=1.571$

$U_{o}=$
$U_{o} / U_{i}=0.918$

53.80, (f) $U_{o}=56.89$,

Fig. 3 Material distributions obtained with standard formulation using the SIMP method
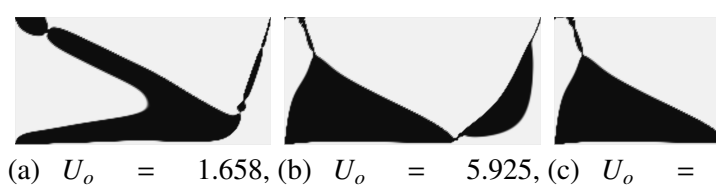

(a) $U_{o}=$

$U_{o} / U_{i}=0.871$

$U_{o} / U_{i}=0.858$

$6.243,($

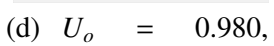

(d) $U_{o}=$
$U_{o} / U_{i}=0.998$

(e) $U_{o}=$
$U_{o} / U_{i}=0.560$

0.49, (f) $U_{o}=52.69$,

Fig. 4 Material distributions obtained with standard formulation using the level set-based method
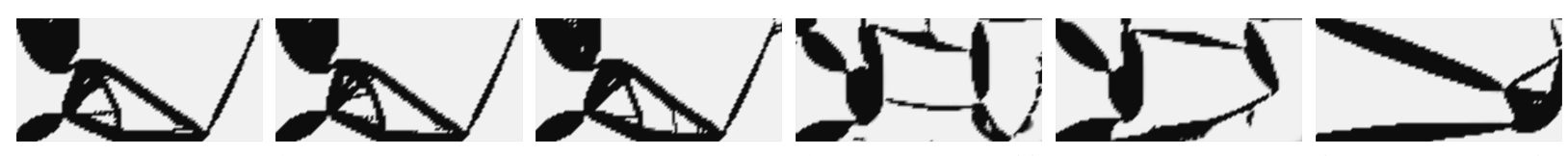

(a) $U_{o}=$
$U_{o} / U_{i}=3.300$ $U_{o} / U_{i}=3.279$

$U_{o} / U_{i}=3.271$

$U_{o} / U_{i}=2.213$

$U_{o} / U_{i}=2.281$

$U_{o} / U_{i}=3.713$

Fig. 5 Material distributions obtained with proposed formulation using the SIMP method

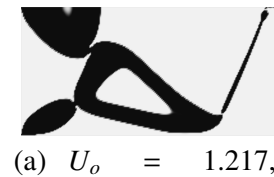

(a) $U_{o}=$
$U_{o} / U_{i}=3.440$

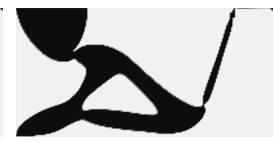

(b) $U_{o}=$
$U_{o} / U_{i}=3.197$

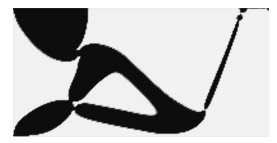

(c) $U_{o}=$
$U_{o} / U_{i}=3.327$

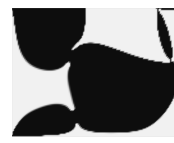

(d) $U_{o}=$
$U_{o} / U_{i}=3.110$

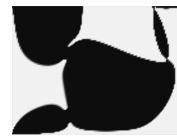

$0.808,($

$U_{o} / U_{i}=3.080$

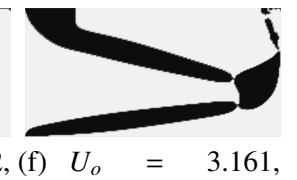

$U_{o} / U_{i}=3.942$

Fig. 6 Material distributions obtained with proposed formulation using the level set-based method

The case (a) results indicate that both formulations give appropriate results. The output displacement of the standard formulation is higher than that of the proposed formulation, but the opposite is true for the GA value, $G A=U_{o} / U_{i n}$. Here, the normalized input displacement $U_{\text {in }}$ is defined as follows:

$$
U_{i}:=\frac{\int_{\Gamma_{i n}} t \cdot u \mathrm{~d} \Gamma}{\int_{\Gamma_{i n}} \sqrt{\boldsymbol{t} \cdot \boldsymbol{t}} \mathrm{d} \Gamma} .
$$

We note that the standard objective function, $F_{s}$, and the proposed objective function, $F_{p}$, are essentially equivalent to $U_{o}$ and $U_{o} / U_{i}$, respectively. The proposed formulation always obtained configurations in which $\Gamma_{u}, \Gamma_{i n}$, and $\Gamma_{o u t}$, are connected, indicating that the use of artificial spring components is not required.

On the other hand, the standard formulation obtained non-connected configurations for cases (d), (e), and (f). We therefore can confirm that the proposed formulation is particularly appropriate for design problems dealing with displacement magnification mechanisms. Note that, of course, the standard formulation provides useful results for many design settings and has been broadly and effectively implemented in many industrial applications. The proposed formulation may be useful in certain sensor design problems, such as for gyrosensors, where reaction forces do not require consideration. 


\section{Conclusions}

This letter proposed a formulation for compliant displacement magnification mechanisms based on the concept of effective energy, that eliminates the need for additional spring components. Numerical examples confirmed the usefulness of the proposed formulation. We showed that the proposed formulation provides appropriate results for compliant displacement magnification mechanisms.

\section{Acknowledgements}

This work was supported in part by JSPS KAKENHI Grant Number 16 K05039.

\section{References}

Bendsøe, M.P., Kikuchi, N., Generating optimal topologies in structural design using a homogenization method, Computer methods in Applied Mechanics and Engineering, Vol.71, No.2 (1988), pp.197-224.

Frecker, M., Ananthasuresh, G., Nishiwaki, S., Kikuchi, N., Kota, S., Topological synthesis of compliant mechanisms using multi-criteria optimization, Journal of Mechanical Design, Vol.119, No.2 (1997), pp.238-245.

Her, I., Midha, A., A compliance number concept for compliant mechanisms, and type synthesis, Journal of mechanisms, Transmissions, and Automation in Design, Vol.109, No.3, pp.348-355.

Howell, L.L., Compliant Mechanisms (2001), John Wiley \& Sons.

Kota, S., Joo, J., Li, Z., Rodgers, S.M., Sniegowski, J., Design of compliant mechanisms, applications to MEMS, Analog Integrated Circuits and Signal Processing, Vol.29, No.1-2 (2001), pp.7-15.

Nishiwaki, S., Frecker, M.I., Min, S., Kikuchi, N., Topology optimization of compliant mechanisms using the homogenization method, Computer methods in Applied Mechanics and Engineering, Vol.119, No.2 (1998), pp.238-245.

Otomori, M., Yamada, T., Izui, K., Nishiwaki, S., Matlab code for a level set-based topology optimization method using a reaction diffusion equation, Structural and Multidisciplinary Optimization, Vol.51, No.5 (2015), pp.1159-1172.

Salamon, B., Midha, A., An introduction to mechanical advantage in compliant mechanisms, Journal of Mechanical Design, Vol.120, No.2 (1998), pp. 311-315.

Sigmund, O., On the design of compliant mechanisms using topology optimization, Journal of Structural Mechanics, Vol.25, No.4 (1998), pp.493-524.

Sigmund, O., Design of multiphysics actuators using topology optimization-part I: One-material structures, Computer Methods in Applied Mechanics and Engineering, Vol.190, No.49 (2001), pp.6577-6604.

Sigmund, O., Morphology-based black and white filters for topology optimization, Structural and Multidisciplinary Optimization, Vol.33, No.4 (2007), pp.401-424.

Svanberg, K., The method of moving asymptotes - a new method for structural optimization, International Journal for Numerical Methods in Engineering, Vol.24, No.2 (1987), pp.359-373.

Wang, F., Lazarov, B.S., Sigmund, O., On projection methods, convergence and robust formulations in topology optimization, Structural and Multidisciplinary Optimization, Vol.43, No.6 (2011), pp.767-784.

Yamada, T., Izui, K., Nishiwaki, S., Takezawa, A., A topology optimization method based on the level set method incorporating a fictitious interface energy, Computer Methods in Applied Mechanics and Engineering, Vol.199 No.45 (2010) pp.2876-2891.

Yamada, T., Yamasaki, S., Nishiwaki, S., Izui, K., Yoshimura, M., Design of compliant thermal actuators using structural optimization based on the level set method, Journal of Computing and Information Science in Engineering, Vol.11, (2011) No.011005. 